\title{
Decay of correlations in the dissipative two-state system
}

\author{
G. Lang ${ }^{1}$, E. Paladino ${ }^{2,1}$, and U. Weiss ${ }^{1}$ \\ 1 Institut für Theoretische Physik, Universität Stuttgart, D-70550 Stuttgart, Germany \\ 2 Istituto di Fisica, Università di Catania \& INFM, Viale A. Doria 6, 95129 Catania, Italy.
}

\begin{abstract}
We study the equilibrium correlation function of the polaron-dressed tunnelling operator in the dissipative two-state system and compare the asymptoptic dynamics with that of the position correlations. For an Ohmic spectral density with the damping strength $K=\frac{1}{2}$, the correlation functions are obtained in analytic form for all times at any $T$ and any bias. For $K<1$, the asymptotic dynamics is found by using a diagrammatic approach within a Coulomb gas representation. At $T=0$, the tunnelling or coherence correlations drop as $t^{-2 K}$, whereas the position correlations show universal decay $\propto t^{-2}$. The former decay law is a signature of unscreened attractive charge-charge interactions, while the latter is due to unscreened dipole-dipole interactions.

05.30.-d, 05.40.+j, 73.40.Gk
\end{abstract}

The simplest model that allows to study the interplay of tunnelling and dissipation is the spin-boson model [1,2]. Despite its simplicity, it exhibits generic features of many complex systems in physics and chemistry and has found widespread applications. It has been adopted to describe diverse systems like the tunnelling of atoms in atomic-force devices [3], or the dynamics of the magnetic flux in a rf-SQUID [4], just to mention a few.

The Ohmic spin-boson model exhibits a dynamical phase transition between coherent and incoherent tunnelling. Both for the expectation value $\left\langle\sigma_{z}(t)\right\rangle$ (the population) and the $\sigma_{z}$ autocorrelation function, the transition from oscillatory to overdamped behaviour was found to occur at a damping strength $K=\frac{1}{2}$ at $T=0$ and zero bias $[5$. Concerning the dynamics at long times, these functions behave quite differently. The factorized system-reservoir initial state for $\left\langle\sigma_{z}(t)\right\rangle$ leads to exponential decay [5, 6], whereas the correlated initial state of the symmetrized equilibrium correlation function implies an algebraic decay $\propto 1 / t^{2}$ [8,9].

The dissipative two-state system (TSS) is described by the spin-boson Hamiltonian

$$
H=H_{0}+\sum_{\alpha}\left[\frac{p_{\alpha}^{2}}{2 m_{\alpha}}+\frac{1}{2} m_{\alpha} \omega_{\alpha}^{2}\left(x_{\alpha}-\frac{c_{\alpha}}{m_{\alpha} \omega_{\alpha}^{2}} \frac{a}{2} \sigma_{z}\right)^{2}\right]
$$

where $H_{0}=-\hbar\left(\Delta \sigma_{x}+\epsilon \sigma_{z}\right) / 2$ describes the isolated TSS with level splitting $\hbar \Delta$ and a bias energy $\hbar \epsilon$. The eigenstates of $\sigma_{z}$ are the two localized states $|R\rangle$ and $|L\rangle$ at positions $\pm a / 2$. The effects of an Ohmic bath are captured by the spectral density $J(\omega)=\left(2 \pi \hbar K / a^{2}\right) \omega e^{-\omega / \omega_{c}}$, where $K$ is a dimensionless damping strength and $\omega_{c}$ is a cutoff for the bath modes. Here we are interested in the scaling limit $\Delta_{r} / \omega_{c} \rightarrow 0$, where $\Delta_{r}=\Delta\left(\Delta / \omega_{c}\right)^{K /(1-K)}$ is kept finite. In this limit, the population $\left\langle\sigma_{z}(t)\right\rangle$ and the $\sigma_{z}$ autocorrelation function are universal functions of the renormalized frequency $\Delta_{r}$, i.e., there is no explicit dependence on $\omega_{c}$.

Recently, there has been considerable interest for observables of the tunnelling operator $\sigma_{x}$ [10,11]. The correlations of $\sigma_{x}$ describe the response of the system to a change in the barrier width or in the barrier height. In atomic force devices, this can be contrived by modulating the distance between surface and tip, and in the rf-SQUID by varying the critical current [4. It was shown that, because of an explicit $\omega_{c}$ dependence, both the expectation value and the autocorrelation function of the bare $\sigma_{x}$ vanish in the scaling limit [12].

In this Letter, we study the correlation function of the polaron-dressed tunnelling operator $\tilde{\sigma}_{x}=U \sigma_{x} U^{-1}$ which has a nontrivial scaling limit. The polaron transformation $U=\exp \left\{-i \sigma_{z} \sum_{\alpha} s_{\alpha} p_{\alpha} / 2 \hbar\right\}$ induces adiabatic displacements $\left\{s_{\alpha}\right\} \equiv\left\{a c_{\alpha} / m_{\alpha} \omega_{\alpha}^{2}\right\}$ of the bath modes during the tunnelling process (cf. Ref. [1] 13]). The dressed tunnelling operator, $\tilde{\sigma}_{x}=|R\rangle\langle L| \exp \left\{-i \sum_{\alpha} s_{\alpha} p_{\alpha} / \hbar\right\}+$ h. c. , transfers the particle from one localized state to the other and simultaneously shifts the bath modes by the displacements $\left\{ \pm s_{\alpha}\right\}$. It is just in this way that coherent oscillations are induced and therefore $\tilde{\sigma}_{x}$ is called the coherence operator.

The quantities of our interest are the equilibrium correlation functions of the position, $C_{z}(t)=\left\langle\sigma_{z}(t) \sigma_{z}(0)\right\rangle_{\beta}-\left\langle\sigma_{z}\right\rangle_{\beta}^{2}$, and of the coherence operator, $C_{x}(t)=\left\langle\tilde{\sigma}_{x}(t) \tilde{\sigma}_{x}(0)\right\rangle_{\beta}$. Our emphasis is put on their asymptotic behaviour for general $K<1$. Using a real-time path integral approach, an exact formal series expression for the symmetrized position correlation function $S_{z}(t)=\operatorname{Re} C_{z}(t)$ has been derived in Ref. [14],

$$
S_{z}(t)=1+\sum_{m=1}^{\infty}\left(-\bar{\Delta}^{2}\right)^{m} \int_{-\infty}^{t} \mathcal{D}_{0,2 m}^{(0)}\left\{t_{j}\right\} \sum_{\left\{\xi_{j}= \pm 1\right\}} G_{0, m} D_{0, m}^{(+)}+Q_{s}(t)-\left\langle\sigma_{z}\right\rangle_{\beta}^{2}
$$




$$
\begin{aligned}
& Q_{s}(t)=-\sum_{n=1}^{\infty} \sum_{m=1}^{\infty}\left(-\bar{\Delta}^{2}\right)^{n+m} \tan ^{2}(\pi K) \int_{-\infty}^{t} \mathcal{D}_{2 n, 2 m}^{(0)}\left\{t_{j}\right\} \sum_{\left\{\xi_{j}= \pm 1\right\}} \xi_{1} \xi_{n+1} G_{n, m} D_{n, m}^{(+)} . \\
& \left\langle\sigma_{z}\right\rangle_{\beta}=-\lim _{t \rightarrow \infty} \sum_{m=1}^{\infty}\left(-\bar{\Delta}^{2}\right)^{m} \tan (\pi K) \int_{-\infty}^{t} \mathcal{D}_{0,2 m}^{(0)}\left\{t_{j}\right\} \sum_{\left\{\xi_{j}= \pm 1\right\}} \xi_{1} G_{0, m} D_{0, m}^{(-)} .
\end{aligned}
$$

Similarly, the respective response function $\chi_{z}(t)=-(2 / \hbar) \Theta(t) \operatorname{Im} C_{z}(t)$ is found as

$$
\chi_{z}(t)=-\frac{2}{\hbar} \sum_{n=1}^{\infty} \sum_{m=0}^{\infty}\left(-\bar{\Delta}^{2}\right)^{n+m} \tan (\pi K) \int_{-\infty}^{t} \mathcal{D}_{2 n-1,2 m+1}^{(0)}\left\{t_{j}\right\} \sum_{\left\{\xi_{j}= \pm 1\right\}} \xi_{1} \xi_{n} G_{n, m} D_{n, m}^{(+)} .
$$

Here we have put $\bar{\Delta}^{2}=\Delta^{2} \cos (\pi K) / 2$. The integration symbol contains the time-ordered integrations over $k$ flips in the negative and $l$ flips in the positive time branch,

$$
\int_{t_{0}}^{t} \mathcal{D}_{k, l}^{(r)}\left\{t_{j}\right\}=\int_{0}^{t} d t_{k+l+r} \int_{0}^{t_{k+l+r}} d t_{k+l+r-1} \cdots \int_{0}^{t_{k+2+r}} d t_{k+1+r} \int_{t_{0}}^{0} d t_{k} \cdots \int_{t_{0}}^{t_{2}} d t_{1} .
$$

In these expressions, the summations over the intermediate diagonal (sojourn) states have already been performed. Further, the $\xi_{j}$ summation takes into account the two possibilities for each off-diagonal (blip) state the system takes. The effect of the bias is in the factors $D_{n, m}^{(+)}=\cos \left(\epsilon \sum_{j=1}^{n+m} \xi_{j} \tau_{j}\right)$ and $D_{n, m}^{(-)}=\sin \left(\epsilon \sum_{j=1}^{n+m} \xi_{j} \tau_{j}\right)$, where $\tau_{j}=t_{2 j}-t_{2 j-1}$ is the length of blip $j$. The bath-induced correlations are expressed in terms of the interaction $S_{p, q}=S\left(t_{p}-t_{q}\right)$, where $S(t)=2 K \ln \left[\left(\hbar \beta \omega_{c} / \pi\right) \sinh (\pi t / \hbar \beta)\right]$. In the equivalent Coulomb gas picture, each factor $\exp \left[-S\left(t_{p}-t_{q}\right)\right]$ represents the interaction of a neutral pair of charges, which we refer to as a dipole. The full intra- and interblip correlations are in the factor

$$
\begin{aligned}
G_{n, m} & =\exp \left[-\sum_{j=1}^{n+m} S_{2 j, 2 j-1}-\sum_{j=2}^{n+m} \sum_{k=1}^{j-1} \xi_{j} \xi_{k} \Lambda_{j, k}\right], \\
\Lambda_{j, k} & =S_{2 j, 2 k-1}+S_{2 j-1,2 k}-S_{2 j, 2 k}-S_{2 j-1,2 k-1} .
\end{aligned}
$$

For $C_{x}(t)$, the procedure is more complicated, since the polaron-dressed operator $\tilde{\sigma}_{x}$ acts on the full system-plusreservoir space. Therefore, we have reconsidered the elimination of the bath modes. We have found that the resulting influence functional can be cast into the standard Feynman-Vernon form at the expense of introducing modified system paths. The findings can be put in simple terms: the system's jumps at times zero and $t$ enforced by the operation of $\tilde{\sigma}_{x}$ do not give rise to bath correlations in the influence functional.

The contributions to $C_{x}(t)$ can be divided into two groups. Either the system jumps from a sojourn to a blip state at time zero (group A) or vice versa (group B). This extra jump is accounted for by the upper index $r=1$ in the integration element (6). For both groups, the system is finally in a blip state. The symmetrized correlation function $S_{x}(t)=\operatorname{Re} C_{x}(t)$ and the response function $\chi_{x}(t)=-(2 / \hbar) \Theta(t) \operatorname{Im} C_{x}(t)$ are found as

$$
\begin{aligned}
S_{x}^{\mathrm{A}}(t)= & \frac{1}{2} \sum_{m=1}^{\infty}\left(-\bar{\Delta}^{2}\right)^{m-1} \int_{-\infty}^{t} \mathcal{D}_{0,2 m-2}^{(1)}\left\{t_{j}\right\} \sum_{\left\{\xi_{j}= \pm 1\right\}^{\mathrm{A}}} G_{0, m}^{\mathrm{A}} D_{0, m}^{(+)}, \\
S_{x}^{\mathrm{B}}(t)= & -\sum_{n=1}^{\infty} \sum_{m=2}^{\infty}\left(-\bar{\Delta}^{2}\right)^{n+m-1} \sin ^{2}(\pi K) \int_{-\infty}^{t} \mathcal{D}_{2 n-1,2 m-1}^{(1)}\left\{t_{j}\right\} \sum_{\substack{\left\{\xi_{j}= \pm 1\right\} \\
\xi_{n}=\xi_{n+1}=-\xi_{n+m}}} \xi_{1} \xi_{n+m} G_{n, m}^{\mathrm{B}} D_{n, m}^{(+)}, \\
\chi_{x}^{\mathrm{A}}(t)= & \frac{1}{\hbar} \sum_{n=1}^{\infty} \sum_{m=1}^{\infty}\left(-\bar{\Delta}^{2}\right)^{n+m-1} \tan (\pi K) \int_{-\infty}^{t} \mathcal{D}_{2 n, 2 m-2}^{(1)}\left\{t_{j}\right\} \sum_{\left\{\xi_{j}= \pm 1\right\}^{\mathrm{A}}} \xi_{1} \xi_{n+m} G_{n, m}^{\mathrm{A}} D_{n, m}^{(+)}, \\
\chi_{x}^{\mathrm{B}}(t)= & \frac{1}{\hbar} \sum_{n=1}^{\infty} \sum_{m=1}^{\infty}\left(-\bar{\Delta}^{2}\right)^{n+m-1} \tan (\pi K) \int_{-\infty}^{t} \mathcal{D}_{2 n-1,2 m-1}^{(1)}\left\{t_{j}\right\} \\
& \times \sum_{\left\{\xi_{j}= \pm 1\right\}^{\mathrm{B}}} \xi_{1} G_{n, m}^{\mathrm{B}} D_{n, m}^{(+)}\left\{\sin ^{2}(\pi K) \xi_{n+1}+\cos ^{2}(\pi K) \xi_{n+m}\right\} .
\end{aligned}
$$

The superscripts $\{\ldots\}^{\mathrm{A}}$ and $\{\ldots\}^{\mathrm{B}}$ indicate that the blip labels are subject to the constraints

$$
\xi_{n+m}=\xi_{n+1} \quad\left(\text { group A), } \quad \xi_{n+m}=-\xi_{n} \quad(\text { group B). }\right.
$$


The interaction factors $G_{n, m}^{\mathrm{A}}$ and $G_{n, m}^{\mathrm{B}}$ differ from the form (7) for $G_{n, m}$ by the removal of the charges at times $t^{\prime}=0$ and $t^{\prime}=t$. We have

$$
\begin{aligned}
& G_{n, m}^{\mathrm{A}}=\exp \left[-\sum_{j=1}^{n+m} S_{2 j, 2 j-1}-\sum_{j=2}^{n+m} \sum_{k=1}^{j-1} \xi_{j} \xi_{k} \Lambda_{j, k}^{\mathrm{A}}\right], \\
& G_{n, m}^{\mathrm{B}}=\exp \left[-\sum_{j=1, j \neq n}^{n+m} S_{2 j, 2 j-1}-\sum_{j=2}^{n+m} \sum_{k=1}^{j-1} \xi_{j} \xi_{k} \Lambda_{j, k}^{\mathrm{B}}\right],
\end{aligned}
$$

where $\Lambda_{j, k}^{\mathrm{A}}$ and $\Lambda_{j, k}^{\mathrm{B}}$ describe the interblip correlations for the modified sequences of charges. If $j, k \neq n+1$ and $\neq n+m, \Lambda_{j, k}^{\mathrm{A}}$ is again given by (8). In all other cases, the interactions of the missing charges in Eq. (8) have to be dropped. For instance, for $j=n+1$, we have $\Lambda_{n+1, k}^{\mathrm{A}}=S_{2 n+2,2 k-1}-S_{2 n+2,2 k}$. There are analogous modifications in $\Lambda_{j, k}^{\mathrm{B}}$ for $j, k=n$ and $n+m$. For instance, we have $\Lambda_{n, k}^{\mathrm{B}}=S_{2 n-1,2 k}-S_{2 n-1,2 k-1}$.

For the value $K=\frac{1}{2}$, the above series can be summed in analytic form using the concept of collapsed dipoles [14]. Putting $K=\frac{1}{2}-\kappa$ with $\kappa \ll 1$, the phase factor $\cos (\pi K) \approx \pi \kappa$ vanishes in the limit $\kappa \rightarrow 0$. In order to have a finite contribution for $K=\frac{1}{2}$, each factor $\cos (\pi K)$ has to be compensated by the $1 / \kappa$ "short-distance" singularity arising from the breathing mode integral of a dipole with interaction $e^{-S(\tau)} \approx\left(\omega_{c} \tau\right)^{-(1-2 \kappa)}$. The combined expression is termed a collapsed dipole, yielding the finite contribution

$$
I\left(K=\frac{1}{2}\right)=\lim _{K \rightarrow 1 / 2} \Delta^{2} \cos (\pi K) \int_{0} d \tau e^{-S(\tau)}=\frac{\pi}{2} \frac{\Delta^{2}}{\omega_{c}} \equiv \gamma .
$$

A collapsed dipole does not interact with other charges and it is insensitive to a symmetric bias factor. In contrast, an odd bias factor in Eq. 16 prevents a dipole from collapsing. However, to give a nonzero contribution, the extended dipole must be free of a $\cos (\pi K)$ factor. Within an extended blip [sojourn] state of length $\tau$ [s], the system may make any number of visits of duration zero to a sojourn [blip] state. This is described by the insertion of a grand-canonical gas of non-interacting collapsed sojourns (CS) [or blips (CB)], yielding a factor $e^{-\gamma \tau / 2}$ [or $e^{-\gamma s}$ ]. Note that there is a multiplicity factor 2 for collapsed blips. As a general rule, an extended sojourn interval, say $s_{k}$, is free of insertions only if the subsequent blip is weighted with a factor $\xi_{k+1}$. In the expression (3), e.g., the first sojourns in each time branch are without insertions. Employing these concepts, the following results for the various correlation functions are obtained. The $\sigma_{z}$ correlation functions are given by [2]

$$
\begin{aligned}
& S_{z}(t)=e^{-\gamma t}-F_{+}^{2}(t)-F_{-}^{2}(t), \quad \chi_{z}(t)=(4 / \hbar) F_{+}(t) e^{-\gamma t / 2}, \\
& F_{ \pm}(t)=\frac{\Delta^{2}}{2 \gamma} \int_{0}^{\infty} d \tau e^{-S(\tau)} f_{ \pm}(\epsilon \tau)\left(e^{-\gamma|t-\tau| / 2} \mp e^{-\gamma(t+\tau) / 2}\right),
\end{aligned}
$$

with $f_{+}(x)=\cos (x), f_{-}(x)=\sin (x)$. A diagrammatic representation of $S_{z}(t)$ and $\chi_{z}(t)$ is displayed in Fig. 11.
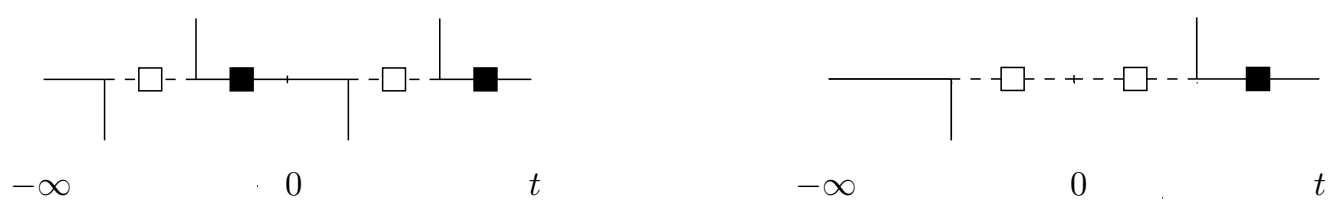

FIG. 1. The diagrams for $\chi_{z}(t)$ (right) and the contribution $Q_{s}(t)$ to $S_{z}(t)$ (left). The full and dashed lines represent sojourns and blips, respectively. An empty box represents the insertion of a CS gas within a blip interval, the full box stands for a CB gas inside a sojourn interval. The upward and downward spikes symbolize charges.

In the asymptotic regime $T=0$ and $t \gg 1 / \gamma$, we find from Eq. (17)

$$
S_{z}(t)=-\left(\frac{4}{\pi \gamma}\right)^{2}\left(\frac{\gamma^{2}}{\gamma^{2}+4 \epsilon^{2}}\right)^{2} \frac{1}{t^{2}}, \quad \chi_{z}(t)=\frac{16}{\pi \hbar} \frac{\gamma^{2}}{\gamma^{2}+4 \epsilon^{2}} \frac{\cos (\epsilon t) e^{-\gamma t / 2}}{\gamma t}
$$

The exponential decay of the response function is due to the grand-canonical sums of collapsed dipoles in each interval (except for the first sojourn). In contrast, the function $S_{z}(t)$ decays algebraically because the first sojourn in the 
positive time branch is free of collapsed blips. Therefore, this interval gets very large and is effectively limited by the overall length $t$. The $1 / t^{2}$ law reflects the bare interaction between the two dipoles displayed in Fig. 目 (left).

Now switch to the $\tilde{\sigma}_{x}$ correlation functions. Since there is one $\cos (\pi K)$ factor more than dipoles in Eq. (10), $S_{x}^{\mathrm{B}}(t)$ vanishes as $K \rightarrow \frac{1}{2}$. Therefore, only group A contributes to the symmetrized correlation function, yielding the damped oscillatory behaviour

$$
S_{x}(t)=\cos (\epsilon t) e^{-\gamma t / 2} .
$$

The corresponding diagram is sketched in Fig. 2 .

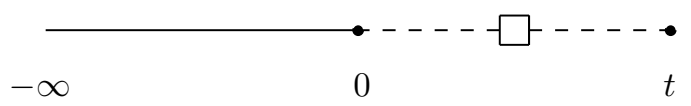

FIG. 2. The diagram describing $S_{x}(t)$. The bullets mark transitions which are free of bath correlations because of the modified influence functional.

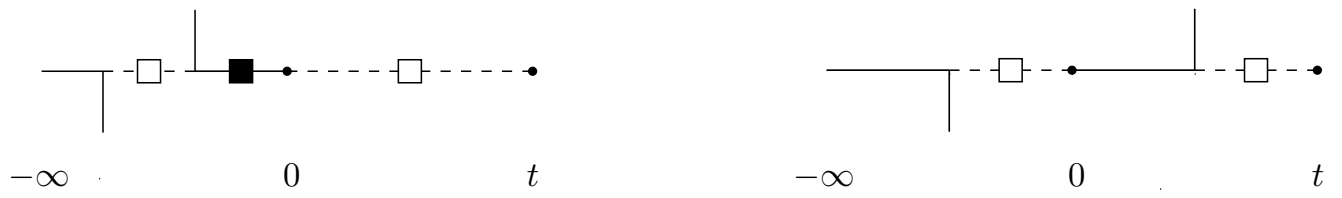

FIG. 3. The contribution of group A (left) and group B (right) to $\chi_{x}(t)$. Each diagram has only one extended dipole.

For the response function, the contributions of both groups are shown in Fig. 3. The expressions are combined to

$$
\chi_{x}(t)=(2 / \hbar)\left[\cos (\epsilon t) F_{+}(t)+\sin (\epsilon t) F_{-}(t)\right] .
$$

At $T=0$ and times $t \gg 1 / \gamma$, we find from Eq. (21)

$$
\chi_{x}(t)=\frac{8}{\pi \hbar} \frac{\gamma^{2}}{\gamma^{2}+4 \epsilon^{2}} \frac{1}{\gamma t} .
$$

The algebraic decay law arises from the contribution of group B. According to the above rule, the sojourn interval in the positive time branch is free of a CB gas factor and therefore is effectively very large for $t \gg 1 / \gamma$. The $1 / t$ law in Eq. (22) is simply the signature of the bare intra-dipole interaction, $e^{-S(t)} \propto 1 / t$ for $K=\frac{1}{2}$.

For general $K$, it is not possible to sum the series for the $\sigma_{z}$ and $\tilde{\sigma}_{x}$ correlation functions in analytic form. Nevertheless, it is possible to extract the long-time behaviour of the correlations. The essential modifications concern the CB and CS gas factors inserted in a given interval. For $K \neq \frac{1}{2}$, the dipoles are no longer collapsed and thus the respective grand-canonical sum can not be performed in analytic form. However, because of the alternating sum, the charges which are partitioned off by the weight factors $\xi_{k+1}$ form clusters of effective length $1 / \Delta_{r}$.

Consider first the $\sigma_{z}$ correlation functions at asymptotic times $t \gg 1 / \Delta_{r}$. For $\chi_{z}(t)$, there is a single neutral cluster surrounding the origin of the time axis. Therefore $\chi_{z}(t)$ decays exponentially. In $S_{z}(t)$, we have a neutral cluster in each time branch. Since in both branches the initial sojourn is free of insertions (see above rule), the two clusters are near the origin and near $t$, respectively. The correlations between the two neutral clusters are dominated by the bare dipole-dipole interaction which is $-2 K / t^{2}$ at $T=0$. The interaction has a universal power-law form which is independent of the coupling strength. The power 2 is a characteristic feature of Ohmic dissipation. The dipole moment emerges to coincide with the static susceptibility [8]. In the end, we have

$$
S_{z}(t)=-2 K\left[\hbar \chi_{z}^{(0)} / 2\right]^{2} \frac{1}{t^{2}} .
$$

In the frequency domain, Eq. (23) corresponds to the generalized Shiba relation [8] for the spectral function, $S_{z}(\omega \rightarrow 0)=2 \pi K\left[\hbar \chi_{z}^{(0)} / 2\right]^{2}|\omega|$.

Next, consider the $\tilde{\sigma}_{x}$ correlation function for $t \gg 1 / \Delta_{r}$. In group A, there is only a single neutral cluster near to the origin of the time axis. Hence, both $S_{x}^{\mathrm{A}}(t)$ and $\chi_{x}^{\mathrm{A}}(t)$ decay exponentially at asymptotic times. In group B, 
we have a charged cluster in each time branch, satisfying overall neutrality. Again, as a result of the above rule, the clusters are situated near the origin and near $t$ and therefore are roughly separated by an interval of length $t$. The clusters interact with unscreened charge-charge interaction $e^{-S(t)} \propto t^{-2 K}$. This interaction directly determines the long-time behaviour of $S_{x}^{\mathrm{B}}(t)$ and $\chi_{x}^{\mathrm{B}}(t)$. The contributions of group B predominate over the exponential contributions of group A for $t \gg 1 / \Delta_{r}$. Thus we find the asymptotic behaviour

$$
S_{x}(t) \propto e^{-S(t)} \propto t^{-2 K}, \quad \chi_{x}(t) \propto e^{-S(t)} \propto t^{-2 K} .
$$

We note that the prefactor in the algebraic decay law for $S_{x}(t)$ vanishes accidentally as $K \rightarrow \frac{1}{2}$. The results 24 ) have been indicated numerically in Ref. [11]. The slow decay of $\chi_{x}(t)$ for $K<\frac{1}{2}$ implies that the static susceptibility

diverges algebraically, $\propto T^{2 K-1}$. Interestingly, this regime coincides with the coherence regime for the population $\left\langle\sigma_{z}(t)\right\rangle$ at zero bias [5, 11].

In conclusion, we have given exact formal expressions for the tunnelling or coherence correlations in the dissipative two-state system for general damping strength $K$ and we have presented results in analytic form for the particular case $K=\frac{1}{2}$. The differences in the long-time behaviours of the position and coherence correlations have been illustrated in terms of a simple charge picture.

\section{ACKNOWLEDGMENTS}

E.P. thanks R. Fazio and G. Giaquinta for useful discussions and acknowledges financial support by the INFM under the PRA-QTMD programme. Partial support was provided by the Deutsche Forschungsgemeinschaft (DFG).

[1] A.J. Leggett, S. Chakravarty, A.T. Dorsey, M.P.A.Fisher, A. Garg, and W. Zwerger, Rev. Mod. Phys. 59, 1 (1987); ibid. 67, 725 (1995) [erratum].

[2] U. Weiss, Quantum Dissipative Systems, Series in Modern Condensed Matter Physics, Vol.2, (World Scientific, Singapore, second edition, 1998).

[3] D.M. Eigler and E.K. Schweizer, Nature 344, 524 (1990); A.A. Louis and J.P. Sethna, Phys. Rev. Lett. 74, 1363 (1995).

[4] R. Rouse, S.Han, and J.E. Lukens, 75 Phys. Rev. Lett. 75, 1614 (1995) and references therein.

[5] R. Egger, H. Grabert, and U. Weiss, Phys. Rev. E 55, R3809 (1997).

[6] F. Lesage, H. Saleur, Phys. Rev. Lett. 80, 4370 (1998).

[7] K. Völker, cond-mat/9712080 preprint.

[8] M. Sassetti and U. Weiss, Phys. Rev. Lett. 65, 2262 (1990).

[9] T.A. Costi and C. Kieffer, Phys. Rev. Lett. 76, 1683 (1996).

[10] M. Grifoni, M. Winterstetter, and U. Weiss, Phys. Rev. E 56, 334 (1997).

[11] S. P. Strong, Phys. Rev. E, 556636 (1997).

[12] F. Guinea, Phys. Rev. B, 324486 (1985).

[13] R. Silbey and A. Harris, J. Chem. Phys. 80, 2615 (1984).

[14] M. Sassetti and U. Weiss, Phys. Rev. A 41, 5383 (1990). 